\title{
Working with struggling teams
}

\section{Guy Undrill \& Nathan Gregory}

\begin{abstract}
SUMMARY
Consultant psychiatrists are often called to work in teams that are functioning suboptimally. This is a major challenge, both professionally and personally. This article gives advice and strategies for working with struggling teams. It recommends combining objective-, data- and procedure-driven approaches to technical challenges with 'softer' person-centred and relational strategies matched to the learning needs of the team.
\end{abstract}

\section{DECLARATION OF INTEREST}

None.

Consultant psychiatrists can find themselves in teams that, for a variety of reasons, are struggling: the team may be underperforming in respect of its core functions, it may be suffering from poor morale or it may simply be struggling to survive in a changing organisation. Working in teams that are struggling makes major demands on the leadership skills of psychiatrists. Psychiatric training does not always prepare new consultants for these kind of challenges, which are not uncommon in first posts. We suggest a four-stage process:
1 information-gathering
2 building a guiding coalition
3 action/implementation
4 consolidation.

Before looking at each of these stages in more detail, we consider a typology of struggling teams and offer a health warning.

\section{A typology of struggling teams}

West (2004) describes teams functioning in terms of task effectiveness, mental health and viability. Each of these can be impaired. Although teams rarely struggle on one domain only, it is helpful to think in terms of dimensions of team functioning that can be compromised.

\section{Task effectiveness}

Task effectiveness is subdivided here (following Hawkins 2011) into skills drift and model drift.

\section{Skills drift}

Skills drift results from a variety of causes: inadequate supervision and training are typical, as is a team culture in which reflective practice is devalued. This is because the team (or team manager) usually has more control over training, supervision and reflection than over clinical work and these elements of the workload may be jettisoned in an effort to reduce time pressure. Of the many clinical and managerial skills that can be subject to this kind of drift, teamworking itself is worth a particular mention. When handovers, recordkeeping and team meetings are ineffectual or seen as peripheral to patient care, the team's effectiveness will rapidly degenerate. West (1996) also stresses the importance of task reflexivity - the ability of the team to reflect and act on its objectives, strategies and processes in the context of the organisational and wider operating environment.

\section{Model drift}

Model drift is common when teams take on tasks unrelated to their core function or cease to understand and implement their core function effectively. It is sometimes a function of disengagement from the wider organisation and its goals, which can be a part of a deliberate distancing strategy by the team if they have a strong ethos that they believe is incompatible with that of the wider organisation. It can also happen through lack of management oversight (or by managers loading teams with tasks extraneous to their main function). Lack of managerial oversight is a greater risk where the team is geographically separate from other parts of the organisation. More insidiously, model drift occurs if new members replace old members without an adequate induction, leading to an increasing proportion of the team without a clear understanding of what the team does and why. Two to three years after inception, this can creep in to teams set up by enthusiasts to do a particular task when the first flush of enthusiasm has passed, especially if key figures have moved on.

\section{The mental health of the team}

The mental health of the team incorporates its well-being, health and development. It is signified by important objective measures of team functioning, such as sickness absence and staff turnover. Sometimes, seemingly contingent effects such as a cluster of suicides can have a knock-on effect on a team's mental health. However, such events can also expose an underdeveloped capacity
Guy Undrill is a consultant general adult psychiatrist in ${ }^{2}$ gether NHS Foundation Trust, Cheltenham, UK. Nathan Gregory is a Community Services Manager for Working Age Adults in ${ }^{2}$ gether NHS Foundation Trust. Correspondence DrGuy Undrill, ${ }^{2}$ gether NHS Foundation Trust, Lexham Lodge, Copt Elm Road, Cheltenham GL53 8AG, UK. Email: guy.undrill@doctors.org.uk 
to contain anxiety (Obholzer 1994) or a lack of social reflexivity (the ability of a team to look after its members; West 1996). Factors that can have a negative impact on the mental health of the team generally centre on conflict: too much, too little or the wrong sort.

Too little conflict

Particularly in a multidisciplinary team, a healthy divergence of professional opinion is important for high functioning and the team that always agrees is unlikely to be performing optimally. This can arise in teams that are overcommitted to a single model of working, leading to a situation where teams with other perspectives are routinely denigrated as part of the index team's way of describing themselves: conflict is then experienced at the team's boundaries rather than within it. In more extreme cases, this develops into 'groupthink' within the team (West 2004).

Too much conflict

More commonly, teams experience too much conflict, often along interprofessional lines. One key area of conflict is between team managers and consultants. The ambiguities surrounding power, authority, clinical leadership and operational management that are often present in NHS teams contribute to a 'rich vein of conflict within teams' (Onyett 2003), which is beyond the scope of this article. In purely practical terms, however, when respective roles and responsibilities are unclear or there is a breakdown in trust between manager and consultant, difficulties are often particularly challenging to resolve.

\section{Viability}

West (2004) defines viability as the probability that the team will continue to work together. Viability is threatened by unclear goals, structure and direction, and when a team cannot attend to the health and development of its members. A team that lacks stakeholder support will often wither and die as stakeholders look for other avenues to meet their needs. Once a team ceases to have organisational support it may implode quite rapidly: for example, when the closure of a ward is announced, staff immediately start applying for jobs elsewhere and the unit may cease to be able to provide safe levels of staffing cover sooner than senior managers planned.

\section{Do I really want to do this?}

Turning around a team that is struggling or underperforming can carry high professional and personal costs. Heifetz et al (2009) caution against the myth that teams or organisations need to change because they are broken: they are that way because (some) people want them that way. If you are the person who questions the status quo and upsets the equilibrium of the team, forces within it will certainly push back against you.

\section{Technical and adaptive challenges}

Heifetz et al make the valuable distinction of technical challenges and adaptive challenges. Technical challenges are typically clearly defined with well-described solutions; meeting the challenge means an objective of restoring order and re-orienting people to norms and roles. Adaptive challenges require learning both to define the problem and to deliver a solution. Conflict may need to be brought to the surface as norms are challenged and roles shift. Treating adaptive challenges as technical ones is often considered the key mistake made in most change efforts.

\section{Relationships}

What is at stake in changing how a team works is not adherence to policy or using a particular care pathway: it is loyalties, identities and relationships. Shifts in these domains will involve personal change and loss for the people involved and if you are the person bringing these changes you may not be popular. Hawkins \& Smith (2006) phrase the same problem slightly differently, noting that problems should be addressed within relationships, not individuals, teams or departments. There is often a temptation to blame an individual or a team and to think that by removing the individual or breaking up the team the problem will go away. Hawkins \& Smith caution against this and suggest that focusing on relationships is the way to shift culture in teams.

\section{Assessing the costs}

We would suggest that the first decision that you should make when faced with the challenge of changing how a struggling team works is whether you are sure you really want to do it. If you have a choice, particularly if you are a new consultant or are currently experiencing stress in other parts of your life, think hard about walking away. The personal costs can be high.

\section{The consultant's involvement}

Other than joining a team and finding out about the issues when they start work, there are two specific situations in which consultants can find themselves working with a struggling team. In the first, the consultant is already there and the team's problems have crept in. The best case scenario here 
is that the consultant will have to start by making changes to themselves. These changes may include sacrificing cherished beliefs about themselves, facing things about themselves that they would rather not acknowledge, realigning important values or working on their own failing relationships with members of the team or people with whom the team interface. (The consultant often carries the primary responsibility for interfaces with other teams and can set the tone for how well interfaces between teams work.) This is rarely achieved without sleepless nights. The worst-case scenario is if the problem is the consultant's inability or unwillingness to change.

The second situation is when a consultant has been asked to work with a team already identified as 'a problem'. It is sometimes easier to change a team coming in from the outside. Watch for one snare: sometimes the person offering the job is part of the problem. Your brief is unlikely to give you the remit to move or change that person particularly if they line manage you. The worst-case scenario here is that your manager consciously or unconsciously recognises their role in the problem (and your inability to change it) and you are being set up to fail.

In either situation, whether you want to take the job or feel obliged, think about getting a mentor, preferably in another organisation, with whom one can share problems and ideas.

\section{Beginnings: information-gathering}

If you are a consultant starting with a struggling team, first take time (up to a month) to do some information-gathering without moving to action. Keep notes of conversations that you have.

The information you gather will fall into three broad categories: hard data about the team; perceptions and relationships; and policies, procedures and guidance.

\section{Hard data about the team}

Box 1 lists some of the questions that you might ask when gathering hard data. You may find that one or two initial domains of poor practice represent the tip of the iceberg and that there is more below the surface when you look. Poor data may be being used to conceal poor practice.

\section{Perceptions and relationships}

Gaining information about perceptions and relationships within the team can be harder. If you are already in the team, some of your vision may be clouded by strained relationships. Heifetz et al (2009) suggest taking an objective view of what is happening in the team. Remember as
BOX 1 Questions to ask when gathering hard data about the team

-What is the quality of note-keeping and of patient records?

-What about records of team meetings?

- Has the team completed audits? Look at the results. What else needs auditing?

- Has the team published research? Read it and look at the original data if you can.

- What data is there for activity levels in the team (caseloads, contacts etc.)?

- Are there open complaints?

- In respect of personnel, are there grievances against people in the team or taken out by members of the team?

- How much sick leave is being taken?

- How good is the team's compliance with statutory and mandatory training?

- Look at finances. Is the money coming into and going out of the team adequately accounted for?

well the maxim that most change challenges are adaptive ('soft', relational) rather than technical. Getting this bit of the diagnosis right is critical.

Think in terms of self-perceptions. How does the team see itself? What rituals does the team have to affirm its belief (positive or negative) in what it is doing? How do individual members of the team see things? Is there any evidence of bullying or harassment? What are the stories that team members tell about the team to explain its current situation? Typically these stories blame particular individuals or departments (for example, 'If only we could fire X, everything would be so much easier' or 'Management don't understand the importance of what we're doing') and elide the role of the people in the team for creating the situation. Sometimes the problem is framed as being so big and thorny that nobody could be blamed for not taking it on and sorting it out - a manifestation of the adage that teams work incredibly hard to maintain their status quo.

Also, explore external perceptions (Box 2). There are likely to be some major discrepancies between the stories that you hear. At this stage, listen carefully without passing judgement and remember that all accounts will be partial in some way.

\section{Policies, procedures, guidance}

During your diagnostic phase, also gather policies and procedures pertaining to problem areas, for 
B0X 2 Exploring external perceptions of the team

- How do others teams and other people within the organisation see the team?

- Does the team have links or a reputation beyond the organisation?

- Does the team have formal or informal links outside of the organisation?

- How might agencies with which the team links influence people within your organisation?

- How do you feel when you walk into the team room? What does this tell you?

example, care programme policies. Efforts for change invariably bring conflict, so expect it and be ready for it when it comes. Obtain and read the policies of your trust (England and Wales) or board (Scotland) for likely areas of conflict and local mechanisms for addressing them: of particular importance are grievance policy, sick leave policy and disciplinary policy.

Look out guidance on what the team should be doing, for example, national service frameworks and policy implementation guides. This can be supplemented by informal conversations with teams doing similar work in other parts of the country.

\section{Build a guiding coalition}

Trying to change a failing team on one's own as a consultant psychiatrist is both personally and professionally risky. At the same time as your diagnostic fact-finding, start building a guiding coalition. This should be broad and secure before you move to action. It should closely align strategic goals with operational management tasks and establish a communication channel between senior executives and the consultant and team manager. The group might take the form of a regular team 'recovery meeting' with team consultant, team manager, medical director, human resources director and nursing director. If you don't have senior management buy-in, the rest of the process will be much more difficult and significantly more likely to fail.

Aim for a high level of trust and openness in this group. Conversely, try to avoid having people in the group who you know might work against trust and openness (big egos or people who will damage trust). Work to understand the interests, limitations and loyalties of the people in this group. Make sure that the people in this group know what the issues are and are prepared to prioritise fixing them, and that this includes budgeting for some of the things that you will need to do. Note that the process of aligning strategic and operational goals isn't a one-way process of senior managers telling you what your operational goals are. Ideally, strategy should be modified by this encounter too as information is passed up to senior management. Some authors (for example, Mintzberg 1987) refer to strategy as emergent and crafted in deference to this two-way process.

As well as your guiding coalition, work on other key relationships early on. If there are user or carer groups, start building links with them. Keep lines of communication open with consultant colleagues, both within and beyond the trust or board. For your own sanity, maintain strong relationships outside of work.

In National Health Service teams, the relationship between the consultant and the team manager is crucial. Stay in regular touch and don't allow yourselves to be split.

\section{The action stage}

The point at which you have gathered all of your information and built a guiding coalition is effectively your last chance to get out before things get tough. In particular, if you find yourself in a team where the relationship between you and the manager has irretrievably soured and there is no mutual trust, it may be time for one of you to move on before the team's issues are addressed.

Your team manager (or other managers) may have responsibility for some of the tasks listed below, and the degree to which you are directly involved will vary according to local management arrangements, your personal style and the working relationship you have with your team manager. We recommend that the consultant and the team manager at minimum liaise closely on the following tasks. Although you should not overstep your remit as a consultant, change often involves a certain amount of flexibility and creativity in respect of your own job and it may be appropriate for you to take on more responsibility for some of the tasks listed than you might under normal circumstances.

The tasks described need to be achieved more or less simultaneously. Kotter (1996) suggests that establishing a sense of urgency is the most important part of a successful change effort: keep a high pace of change and aim to have essentially completed the tasks within 3 months.

\section{The technical aspects}

\section{Recruitment}

Teams that are in a bad way often haemorrhage staff, thus increasing the pressure on remaining 
staff to cover clinical commitments; staff become more stressed and more likely to leave in turn. Stop this 'death spiral' by getting the team up to complement. Get a quick fix by using agency staff or secondment from other teams to pull people in. If 'donor' teams resist, the backing of the senior management that you secured earlier on can help to combat objections. If necessary, second people out of the team: this might be to protect competent but less robust members of the team who can come back later, or it might be to work around problems that can't be solved immediately (for example, grievance procedures may take longer to follow than the time you have to work with the team). Temper your efforts to get up to complement with the realisation that it may be best for some people to leave the team. Trying to persuade people to stay who really want to leave won't help anyone. If there are key figures within the team who aren't going to be able to sign up to the changes, offer them an exit strategy that allows them to go quickly with pride and reputation intact as far as is possible: your focus is on this team and you don't want someone outside of the team or the organisation running it down. Make sure that people leaving agree to drop all grievances as part of severance.

\section{Training}

It is rare for a change effort to succeed without a learning component. Use the budget that senior managers agreed during your coalition phase to form a comprehensive, high-quality training programme. Take people out of work for this and don't compromise even when you come under pressure to do so, for example, to meet clinical commitments (this is why you got the senior managers on board). Take up to 20 days in blocks or on a weekly basis. This should cover team-building, statutory and mandatory training and any skills deficits. Aim to bring in national experts and good quality speakers to provide enjoyable, high-status training. Work towards repairing the team's self-belief and promoting high expectations within the team. Empowering the team to remove barriers to change on the ground is crucial: use the training to rewrite the mission, operational policy and care pathways as a group so that the team understands and 'owns' these documents. Box 3 shows Onyett's (2003) useful checklist for developing an operational policy.

The practices of appreciative inquiry (Hammond 1998; Cooperrider 2008) can be helpful at this stage. If there are problems with interfaces between the team and other teams, invite representatives to attend sessions and work on the issues together. Elicit the performance indicators
BOX 3 A checklist of issues for the development of operational policy

\begin{tabular}{lc}
\hline - Values & - Record-keeping and data-bases \\
- Aims & How users and carers access them \\
- Objectives & Policy on confidentiality \\
- Client group & - Agree procedures for carrying and \\
- Access & administering medication \\
Agreed referral services & - Staffing \\
How to refer & Team composition \\
How referrals will be prioritised & Roles \\
Procedures for processing refused & Safety procedure for staff (e.g. what to \\
referrals & do if a staff member fails to return from \\
Agreed response times & a visit) \\
- Assessment & Rotas, including out of hours \\
Risk assessments & - Management and other non-clinical roles \\
Initial interview process & - Accountability and reporting relationships \\
Process for informing the referrer of the & - Interagency relationships: e.g. primary \\
outcome of assessment & care, voluntary sector, health-social \\
- Care coordination procedures & services integration \\
Identifying existing or arranging new & - Meetings: purpose, duration, location, \\
care coordinators & ground rules (e.g. turning off pagers), \\
Procedures for planning and reviewing & chairing, recording \\
care & - Training \\
Evidenced-based interventions available & - Resources available to the team \\
and their application (e.g. relapse & - Ouality assurance, audit and the \\
prevention) & participation of service users \\
Statement on the involvement of carers & \\
- Crisis response & \\
Service available out of hours & (After Onyett 2003: pp. 184-5. \\
Crisis response available & \\
Processes for individual planning for & \\
future crisis response &
\end{tabular}

from the team that they think are relevant and build these in to your data-gathering. Make it clear that you will hold the team to account for their work, but that you will do so in a way that is compatible with clinicians' professional ethos and values. Although you will have to collect some data 'to feed the machine', keep this to a minimum and collect data that people on the ground feel are useful measures of the team's progress. Maintaining ownership of your benchmarks like this can be a useful way of keeping the team learning and being fleet in making further changes.

\section{Staffing}

Use occupational health to get a return-to-work strategy for your long-term sick staff. Resolve grievances: you have already brought on board the human resources director, who will prioritise them.

\section{Improve your data}

Bring in agencies external to the team (and possibly to the organisation) to establish exactly how and 
where the team is struggling. For example, the trust or board care coordination lead might help with a care coordination audit, or the governance lead with a notes audit. Generate robust mechanisms of gathering data about clinical activity. Set targets and share the data and targets with the team.

Patients

Resolve complaints and engage with user/carer groups, especially if they are feeling wronged.

\section{The adaptive aspects}

Create space for loss

Tuckman's (1965) well-known model of team development (forming, storming, norming and performing) sometimes has a fifth stage added: mourning (Tuckman \& Jensen's 1977 article refers to this as adjourning). The entry point to the change cycle is usually the mourning stage. However unproductive, unhappy or dysfunctional a team has become, it has a history, a shared culture and values. At some point, these embodied hope and meaning for the people working there. If things are changing, space needs to be given for the team to mourn its loss. If loss isn't worked through, it can be a barrier to a new team forming. As a consultant coming into a team where some members are mourning, the main intervention should be simple reflective listening and validation to ensure that the 'old' team is recognised for its achievements and work.

\section{Build trust}

One of the main differences between better performing teams and less well performing teams is the degree of trust within the team. Teams that trust each other work through disagreements and can experiment and take more confident, less defensive decisions about risk. Where trust is an issue, rumours abound, people skirt around issues and defensive practice is the norm. There may be marked variations in practice within the team. There will also be marked information asymmetries, with different people or subgroups having different information or opinions that aren't shared openly with the whole team. Where trust has completely broken down, members of the team may use grievance procedures. However, their time-consuming and procedural nature can severely reduce your scope for quick action and anything you can do to support staff in resolving issues before they get to this extreme of loss of trust is time well spent. Build trust by being consistent with messages within and outside of the team. Make sure that your own 'unofficial' communications (e.g. pre- and post-meeting chats with colleagues) are in line with your 'official' communications. Trust is often also an issue between teams. Encourage your team to take the leap of faith of 'trusting first' in their relations with other teams.

If trust is low and the team's previous achievements are not noticed, team members may be unhappy and may be actively seeking other work. Changing the atmosphere in the team is important. Keep a high profile and visibility in the team to try to arrest this process: if people have objections, hear them out in private.

Fear and anxiety Senge (1999) says that most of what passes for teamwork is 'the smooth surface, the apparent absence of any problems... everyone sits quietly through the team meeting, then talks about how they feel... over a drink that evening' (p. 241). It is important that powerful unspoken issues should be addressed and not left to fester, but remember that the reason people aren't talking about them is fear and anxiety. Unless you have done the groundwork of building trust to allow people to speak frankly about threatening issues, it isn't going to happen.

Fear and anxiety may be about the specifics of the change itself or about the inevitable uncertainty and ambiguity that a change effort brings. Holding anxiety about ambiguity (without prematurely closing it down) is very much a consultant function in this situation.

Addressing negativity Closing down negativity by having open, trusting conversations about the real issues is possibly the hardest part of the task of changing a team's culture. It often feels like a distraction from real work (seeing patients, correspondence, meetings, etc) and is emotionally difficult, so easily slips to the bottom of the to-do list. In fact, addressing negativity is often the most important part of the task. It will usually involve seeking out difficult conversations and then being unreactive to your own negative affect. One may be heavily criticised (probably not to one's face), or other people in the organisation will be criticised in your presence. The temptation to shoot the messenger when criticism is being delivered in an affect-laden way is often overwhelming and can prevent you from hearing the important points buried in the attack. Often the team's view of their performance will be very different to that of senior management: neither side will be entirely correct, although opinions may be polarised and held with a high degree of conviction and associated affect. Arguing back and wanting to defend the side with whom you identify more will bog things down. 
When caught in the crossfire, a useful maxim is to side with management whenever you can (deliver the strategic goals) and side with your staff whenever you should (protect them from blame or persecutory management). Don't compromise your integrity for either side: it is difficult to come back from a breach of trust.

\section{Change the identity of the team}

External and internal perceptions of the team may need to shift. Make sure that the team is made of aware of their achievement every time they do something to be proud of. Be alert not just to the achievement of the milestones that you think are important, but also to 'unanticipated accomplishments' (Senge 1999). Think about marketing strategies: publish stories in the trust or board newsletter, start a website, run a competition for a new name, get some stories in the local paper.

In changing the identity of the team, getting 'quick wins' is often considered crucial (Kotter 1996). Quick wins are important, both for the team's self-perception and for external stakeholders (the senior managers who signed up to supporting you through this). But driving too hard for quick wins can create its own problems. Van Buren and Safferstone (2009) identify a paradox where the management behaviours most likely to bring quick wins are also the behaviours most likely to undermine the overall change effort. They identify five such behaviours:

- focusing too much on detail;

- reacting negatively to criticism;

- intimidating others;

- jumping to conclusions; and

- micromanagement.

Experiment and make mistakes. Your plan for the team is today's best guess. Don't be afraid to tear it up tomorrow.

\section{Difficulties}

Most members of underperforming teams are capable people doing their best in a difficult situation. It's good to remember this for a variety of reasons, not least of which is that it will reduce your own stress. Avoid the easy temptation to think of the whole team as a 'nest of vipers' and instead try to approach people who may be kicking out at you or the organisation with compassion. Organisational problems are essentially clashes of values played out in interpersonal terms and if you fix the organisational problem you often create the conditions for the personal animosity to begin to fade (although the interpersonal issues will need attention in themselves along the way). However, in the short term, if you are attempting change, flak will come your way.

Occasionally, there will be one or two team members who genuinely can't or won't change and whose presence may be destructive to the team as a whole, and you may be unable to completely change the culture of the team unless they leave. Possible solutions include secondment out, performance management, attendance management, occupational health, redundancy and addressing professional registration.

Some people will make this difficult for you by taking out grievances or using trust or board policies to accuse you of being a racist/sexist/ bullying incompetent. At this point you will need all of the support you can get from your guiding coalition and from your own personal supports. If you find yourself getting stuck or stressed, go to your mentor or re-evaluate your decision not to have one.

\section{Locking the change in}

Getting to the point where the team is functioning well, fully staffed and doing good work with a full caseload can feel like the end. Much management literature (for example: Kotter 1996; Senge 1999) suggests that this is a key danger period: until changes are locked into the culture of the team, they remain fragile. Culture is powerful and the former ways of the team can reassert themselves unless new habits are actively nurtured and consolidated. Kotter (1996) suggests that at this stage, keeping focus on clarity of shared purpose and continuing to learn is vital. Use training and away days to return to the team's mission statement, to reconnect with its values and to review how its work is measured. Teams (and people) develop in the direction that they study. Are the metrics that you started with the right ones? What could you audit differently? Linking with other teams in your region in informal networks and at "brag and steal' events can be helpful.

\section{References}

Cooperrider DL, Whitney D, Stavros JM (2008) Appreciative Inquiry Handbook. For Leaders of Change (2nd edn). Berrett-Koehler.

Hammond S (1998) The Thin Book of Appreciative Inquiry. St Luke's Innovative Resources.

Hawkins P (2011) Systemic Team Coaching. Kogan Page.

Hawkins P, Smith N (2006) Coaching, Mentoring and Organisational Consultancy. Open University Press.

Heifetz R, Grashow A, Linsky M (2009) The Practice of Adaptive Leadership. Tools and Tactics for Changing Your Organization and the World. Harvard Business Press.
MCO answers

1 c 2 e 3 b 4 b 5 e 
Kotter J (1996) Leading Change. Harvard Business School Press.

Mintzberg H (1987) Crafting strategy. Harvard Business Review 65 : 64-75.

Obholzer A, Roberts V (1994) The Unconscious at Work: Individual and Organizational Stress in the Human Services. Routledge.

Onyett S (2003) Teamworking in Mental Health. Palgrave MacMillan.

Senge P, Kleiner A, Roberts C, et al (1999) The Dance of Change. The Challenge of Sustaining Momentum in Learning Organisations. Nicholas Brealy.
Tuckman B (1965) Developmental sequence in small group. Psychological Bulletin 63: 384-99.

Tuckman B, Jensen K (1977) Stages of small group development. Journal of Group \& Organisational Studies 2: 419-27.

Van Buren ME, Safferstone T (2009) The quick wins paradox. Harvard Business Review 87: 54-61.

West MA (1996) Reflexivity and work group effectiveness. A conceptual integration. In Handbook of Work Group Psychology (ed MA West): 555-79. John Wiley.

West MA (2004) Effective Teamwork (2nd edn). BPS Blackwell.

\section{MCOs}

Select the single best option for each question stem

\section{When starting with a struggling team,} before moving to the action stage information should be gathered for:
a up to 1 week
b up to 2 weeks
c up to 1 month
d up to 3 months
e up to 6 months.

2 A guiding coalition should contain:

a team manager and team consultant

b team manager, team consultant and team members

c people with big egos who might work against trust and openness

d team manager, team consultant, service users and carers e team manager, team consultant, human resources director, medical director and nursing director.

3 According to Kotter (1996), recruitment, training, staffing, improving data and resolving conflicts should be completed within:

a 4 weeks

b 3 months

c 6 months

d 9 months

e 12 months.

4 Most of the management literature identifies the key danger period as:

a changing the teams identity

$\mathrm{b}$ locking the change in

c building a guiding coalition d establishing a sense of urgency

e recruiting staff quickly.

5 Cutting down negativity is the hardest part of changing the team's culture. An important aspect of this task is:

a seeking difficult conversations and being unreactive to your own negative affect

b not shooting the messenger when criticism is delivered

c not arguing back and defending your position

d siding with management whenever you can, siding with staff whenever you should

e all of the above. 